\title{
Caging the Blob: Using a Slime Mold to Teach Concepts about Barriers that Constrain the Movement of Organisms
}

\author{
Author(s): Cindy E. Bohland, David G. Schmale III , Shane D. Ross \\ Source: The American Biology Teacher, 73(9):537-541. 2011. \\ Published By: National Association of Biology Teachers \\ URL: http://www.bioone.org/doi/full/10.1525/abt.2011.73.9.6
}

BioOne (www.bioone.org) is a nonprofit, online aggregation of core research in the biological, ecological, and environmental sciences. BioOne provides a sustainable online platform for over 170 journals and books published by nonprofit societies, associations, museums, institutions, and presses.

Your use of this PDF, the BioOne Web site, and all posted and associated content indicates your acceptance of BioOne's Terms of Use, available at www.bioone.org/page/terms of use.

Usage of BioOne content is strictly limited to personal, educational, and non-commercial use. Commercial inquiries or rights and permissions requests should be directed to the individual publisher as copyright holder. 


\section{IN QUIRY \&} INVESTIGATION
Caging the Blob: Using a Slime Mold to Teach Concepts about Barriers that Constrain the Movement of Organisms

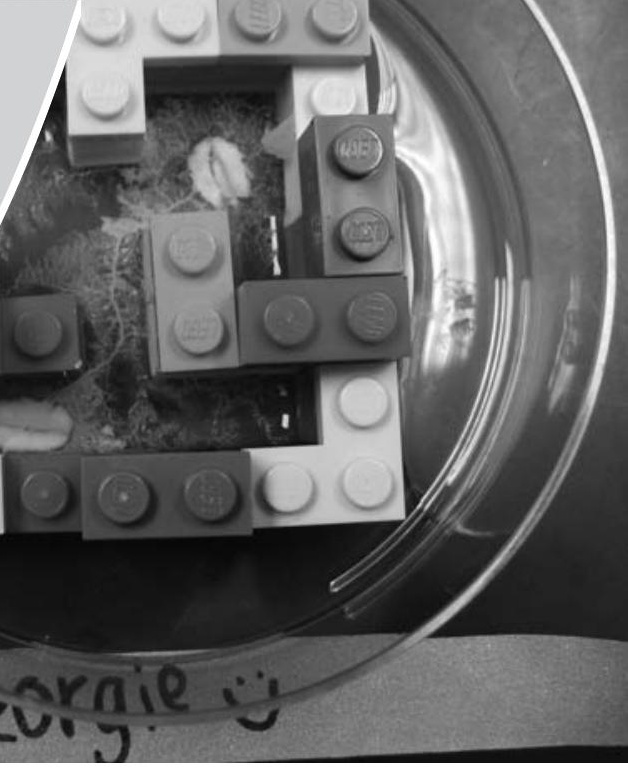

\begin{abstract}
Few laboratory exercises are designed to teach biology students about barriers that may constrain the movement of organisms. We describe a unique inquirybased exercise involving Lego mazes (the barrier) and the plasmodial slime mold, Physarum polycephalum (the organism). During guided inquiry, students construct mazes using Lego brand building blocks and the slime mold is allowed to "navigate" through the maze and "respond" to the barrier. Students then generate and test hypotheses about the movement of the slime mold in response to different barriers in the open-inquiry phase of the investigation.
\end{abstract}

Key Words: Microbe; ecology; atmosphere; barrier; maze; pathogen; transport.

Organisms move from one place to another through land, water, and air. Understanding the ways in which organisms utilize land, water, and air to move from place to place is essential knowledge in studying ecosystems. Sometimes, a population of organisms moving into a new space causes diseases to other organisms or damage to the ecosystem. For example, the zebra mussels that moved through ballast water into the Great Lakes in the 1980s have caused extensive ecological and economic damage (Miller \& Spoolman, 2009). The creation of barriers to prevent the movement of exotic or disease-causing species seems a reasonable solution to this problem. In the case of preventing the movement of organisms through ballast water, ships are advised to take measures to avoid transporting exotic species in ballast water, such as exchanging water only in designated zones (Grodowitz, 2002). This creates a barrier to the movement of aquatic organisms, but only if every ship always follows these voluntary compliances.

The creation of barriers to prevent the movement of exotic or disease-causing organisms becomes an even more complex issue when organisms use air to get around. Many plant diseases are caused by microorganisms that may travel over great distances in the atmosphere. The Irish Potato Famine of the 1840s, a devastating episode in history that resulted in more than a million deaths, was caused by an airborne plant pathogen known as Phytophthora infestans (Schumann \& D'Arcy, 2000). This fungus-like organism produces small lemon-shaped spores that can be transported through air to healthy potato fields (Aylor et al., 2011). If scientists can understand how plant pathogens travel through the air, they can find ways to control their movement and minimize their damage to the crops we eat. This involves understanding barriers to their movement (Isard $\&$ Gage, 2001). For example, a mountain range might create a barrier, effectively isolating a population of microorganisms, or other factors, such as severe weather (e.g., hurricanes) might diminish barriers and accelerate the spread of a potentially devastating disease to crop plants across the country (Isard et al., 2005). Subtler atmospheric phenomena, perhaps due to the long-term effects of global climate change, can also lead to a dynamic landscape of "invisible" atmospheric barriers (Lekien \& Ross, 2010), leading to changes in cyclic patterns of large-scale microorganism movement.

In this two-part inquiry-based lab, students use slime molds to understand the effects of barriers on the movement of organisms. During guided inquiry, students learn to work with the plasmodial slime mold Physarum polycephalum and construct mazes using Lego brand blocks as a barrier. During open inquiry, students design their own experiments to test barriers they predict will be effective in controlling the movement of the slime mold. This investigation is a fun way to introduce the scientific method and can be integrated through content areas such as the cell, ecology, and behavior of organisms. All of the National Science Education Standards for inquiry are addressed (National Academy of Sciences, 1996, pp. 176-177), giving students opportunities to develop skills in all areas of scientific investigation.

\section{O Slime Molds}

Many of us are familiar with slime molds through the seemingly overnight appearance of yellow or orange "slime" on a mulch pile or in a garden bed. Slime molds are not actually molds or even fungi; rather,

The American Biology Teacher, Vol. 73, No. 9, pages 537-541. ISSN 0002-7685, electronic ISSN 1938-4211. (O2011 by National Association of Biology Teachers. All rights reserved. Request permission to photocopy or reproduce article content at the University of California Press's Rights and Permissions Web site at www.ucpressjournals.com/reprintinfo.asp. DOI: 10.1525/abt.2011.73.9.6 
they are classified as amoeboid protozoans. This exercise makes use of a plasmodial slime mold known as Physarum polycephalum. The plasmodium is a streaming packet of cytoplasm containing many nuclei. In the plasmodial stage, $P$. polycephalum exhibits an amoeboid type of movement and is known to respond to a variety of environmental stimuli, including light, physical barriers, food sources, and chemical repellants (Nakagaki et al., 2000, 2007; Adamatzky, 2010; Latty \& Beekman, 2010). This response can be easily observed with the naked eye as the plasmodium grows, creating new pseudopodia and thickening tubes in some areas and shrinking back pseudopodia and allowing tubes to die in other areas (tubes that were once alive appear white rather than yellow). As more materials are exchanged between two parts of a tube, the tube thickens and is reinforced. As fewer materials are exchanged, the tube thins and eventually dies (Nakagaki et al., 2007).

Slime molds have been used in several diverse fields of scientific research. Ecologists have used slime molds to study optimal foraging strategies (Latty \& Beekman, 2010), and computer scientists have used slime molds to build a robot controlled by their movement (Knight, 2006). The activities described here were inspired by two clever experiments that demonstrate the "primitive intelligence" of P. polycephalum. Nakagaki et al. (2000) placed P. polycephalum in a maze created by cutting out a pattern of plastic film. They showed that after filling the maze, P. polycephalum shrinks its plasmodium so that it connects two food sources placed at two ends through the shortest distance of the maze (a time-lapse video of the experiment can be viewed at http://www.youtube.com/watch?v=F3z_mdaQ5ac). Tero et al. (2010) used $P$. polycephalum to model the Tokyo rail system. By placing food in areas of concentrated human populations and by shining light to restrict growth to model areas of natural barriers (mountains, lakes, and oceans), they showed that P. polycephalum forms an efficient network of "rail lines." The design of both of these experiments is contingent upon the ability of $P$. polycephalum to sense and respond to barriers in its environment.

\section{○ Barriers}

There are three general types of barriers to the movement of organisms: static, invisible, and dynamic. Static barriers are solid, physical entities. In the guided-inquiry part of this experiment, Lego blocks are used as static barriers to impede movement. The maze experiment described by Nakagaki et al. (2000) used plastic film as a static barrier. The rail-system experiment described by Tero et al. (2010) used light as an invisible barrier (slime molds grow away from light). The term "dynamic" describes barriers that are changing; for example, solid barriers can move, either slowly and smoothly, or can be added or removed abruptly. Barriers can also be both dynamic and invisible; for instance, an area can be light at one time, then dark at another time. Of course, in nature, all three of these types of barriers exist at once and organisms that move must have mechanisms that allow them to sense and respond in a way that maximizes survival and reproduction. In the open-inquiry part of this experiment, students choose, design, and create a barrier to impose on P. polycephalum.

\section{$\bigcirc$ Learning Objectives}

Through this lab experience, the student will:

1. Describe the importance of barriers in constraining the movement of organisms, particularly in the context of pathogens and invasive species.
2. Evaluate the effect of static barriers on the movement of P. polycephalum and relate these movements to the concept that living things sense and respond to their environment.

3. Discuss the question of when "sense and respond to the environment" becomes "primitive intelligence" and create a definition of intelligence.

4. Design a controlled experiment that collects quantitative data to test the effects of a new static, invisible, or dynamic barrier on the movement of $P$. polycephalum.

5. Analyze data and make appropriate conclusions based on the data of each experiment.

6. Evaluate the experimental design of each lab group and make suggestions to improve control groups, constants, and collection of data.

7. Generate a list of new questions that arise after each experiment and consider new experiments to test these new questions.

\section{$\bigcirc$ Guided Inquiry}

\section{Materials (per group)}

- Assorted Lego blocks - long, thin pieces $(1 \times 6$ and $1 \times 8$ bricks $)$ to construct the outside of the maze; shorter, thin pieces to construct the interior of the maze $(1 \times 1,1 \times 2,1 \times 3,1 \times 4$ bricks $)$; corner plates to connect the outside of the maze $(1 \times 2 \times 2)$; flat plates to connect the interior pieces and help hold the maze together. The number and exact length of each piece will depend on each group's maze design. Custom orders of Lego bricks can be made through the company's website. A class set of reusable Legos can be purchased for about $\$ 50$.

- Molten 2\% water agar, autoclaved (2 grams of plain agar per $100 \mathrm{~mL}$ distilled water)

- Petri dishes (standard round $100 \times 20 \mathrm{~mm}$ dishes work well for the maze experiment; square dishes are convenient, but not necessary, for the open-inquiry phase)

- Sterile pipette and forceps

- 70\% isopropyl alcohol

- Parafilm and aluminum foil

- Quaker old-fashioned oats, uncooked (these do not need to be sterilized; the slime mold will eat the bacteria on them)

- Culture of P. polycephalum growing on oats (it is convenient to order sclerotia; Carolina Biological DH-156190) since P. polycephalum will keep indefinitely in that state. Active plasmodia can also be ordered (Carolina Biological DH-156193). Subcultures will need to be prepared either way. To subculture, remove a $P$. polycephalum-covered oat and place culture-sidedown on a plate with $2 \%$ agar. Sprinkle with a few fresh oats. Each student group will need a plate with at least five oats covered in active plasmodia.)

\section{Methods}

1. Construct a maze that will fit in the Petri dish. Disinfest the maze by spraying it down with alcohol and wiping it with a paper towel

2. Pipette $2 \%$ water agar into the maze to cover the bottom. Allow to solidify.

3. Use sterile forceps to place 5 or 6 oats colonized with P. polycephalum (culture side down) throughout the maze (larger mazes will require more oats to cover). 
4. Seal the sides of the plate with Parafilm and cover in foil.

5. Incubate at $25^{\circ} \mathrm{C}$ for about 24 hours.

6. After P. polycephalum has filled in all parts of the maze, remove the inoculated oats with sterile forceps.

7. Place fresh oats at the two ends of the maze. Reseal the plate and cover with aluminum foil.

8. Incubate at $25^{\circ} \mathrm{C}$ in the dark for another 24 hours.

9. Observe the growth of the P. polycephalum for the next few days. Has the plasmodium shrunk back from the dead ends? Is the plasmodium connecting the two food sources? If there was more than one route from one oat to the other, did the plasmodium connect the two oats through the shortest distance?

\section{Questions}

1. After 2 days of growth, did P. polycephalum connect the oats through the shortest distance?

a. Can you provide evidence through quantitative data?

b. Does this mean that the Lego bricks were an effective barrier?

2. What other observations did you make after 2 or 3 days of growth? Provide pictures or quantitative data.

3. Why is it important that P. polycephalum be able to sense and respond to barriers?

a. How would the behavior of limiting growth to the shortest distance between the oats be the best behavior for survival?

b. In what situations would finding ways around the barrier be beneficial to survival?

4. Find and describe an example of how a human-imposed barrier has limited the spread of a plant, animal, or human pathogen.

5. Find and describe an example of a plant, animal, or human pathogen that barriers have not been effective at stopping.
6. Do you think P. polycephalum exhibits intelligence? Define intelligence and defend your answer.

\section{Safety Notes}

Physarum polycephalum is nonpathogenic and safe for use in the biology classroom. While working with slime molds, students should practice sterile technique to prevent contamination and growth of unknown microorganisms that could possibly be harmful. Students should not be allowed to open a contaminated plate. Students should also adhere to general good laboratory practices. All students should wash their hands at the conclusion of the lab, and food and drink should not be permitted in the laboratory. At the conclusion of the experiment, the slime mold can be disposed of by soaking Petri dishes in a solution of $20 \%$ bleach or by autoclaving. The dishes can then be placed in the general trash.

\section{O Open Inquiry}

For this part of the experiment, students design their own experiments to test the effectiveness of a new static, invisible, or dynamic barrier on the growth of P. polycephalum. Students are encouraged to use different, unique barriers (e.g., chemicals, lack of moisture, etc.), but they are not permitted to intentionally kill the slime mold. During this phase of the lab, the teacher plays an important role in helping students think about experimental design. Students will need help in thinking about controls and how data will be collected. Asking guiding questions will help them evaluate their experimental design. Usually, group discussion following a teacher's question will lead the students to figure out the solution on their own rather than having the teacher tell them what to do.

Table 1 shows the design of an experiment using lack of moisture as a barrier. The group of students decided to use dry paper towels to create the dry areas (they learned from their previous experiment that $P$. polycephalum will grow over the plastic of a Petri dish). The

\section{Table 1. Design of a group experiment to examine the lack of moisture as a barrier to the movement of the slime mold Physarum polycephalum. Students were provided with square Petri dishes. Barriers were created either by cutting a square piece out of the center (exterior agar) or by cutting a strip of agar spanning the width of the Petri dish (divided agar).}

\begin{tabular}{|l|l|l|}
\hline Experimental Design & \multicolumn{1}{|c|}{ Purpose } & Explanation \\
\hline Exterior agar/dry center & Experimental plate & Will the slime mold cross a dry paper towel or grow around it? \\
\hline Divided agar/dry center & Experimental plate & Will the slime mold cross a dry paper towel? \\
\hline All agar & Control plate & $\begin{array}{l}\text { Demonstrates the pattern of growth from one oat to another } \\
\text { under optimal conditions. }\end{array}$ \\
\hline All dry paper towel paper towel & Control plate & $\begin{array}{l}\text { Demonstrates that moisture, not something else about } \\
\text { the paper towel, is the factor preventing growth. Growth is } \\
\text { expected on this plate. }\end{array}$ \\
\hline Exterior agar/wet center & $\begin{array}{l}\text { Demonstrates that the slime mold cannot grow without moisture. } \\
\text { No growth is expected on this plate. }\end{array}$ \\
\hline Divided agar/wet center & $\begin{array}{l}\text { Control plate } \\
\text { is a ledge of agar or something else. Growth is expected across } \\
\text { the center. }\end{array}$ \\
\hline
\end{tabular}


students had to think carefully about experimental design and control groups. For each plate, they placed two inoculated oats on opposite sides of a Petri dish.

\section{Results \& Conclusions}

Students should be able to replicate Nakagaki et al.'s (2000) results, demonstrating that slime mold will connect the shortest distance between two oats (Figures 1 and 2 show student results of two maze experiments). Although quantifying this claim may seems obvious, some students will need to be prompted to take measurements. Simply asking the student groups "How could you convince somebody else that the slime mold did find the shortest distance?"

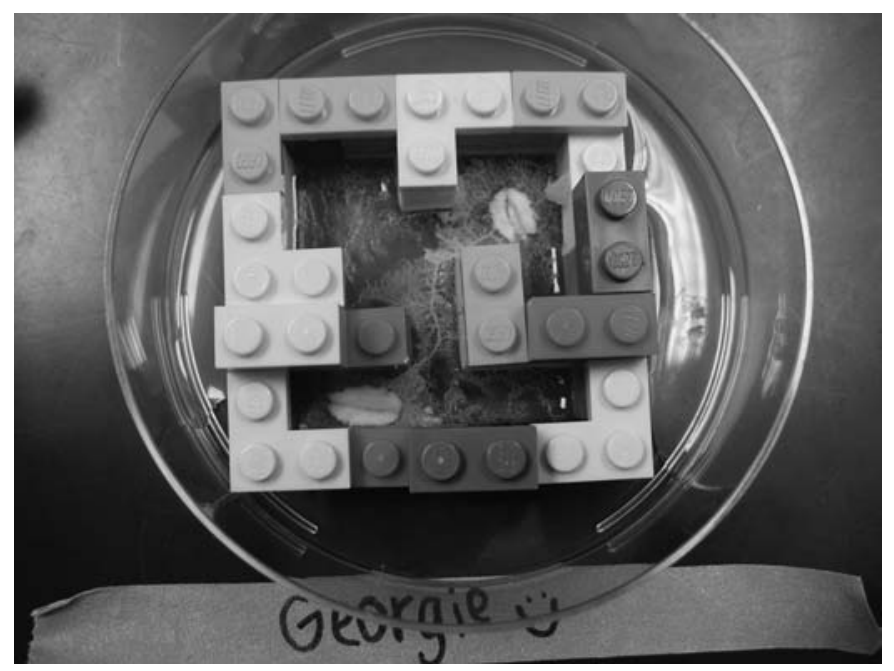

Figure 1. Results from a maze experiment showing the plasmodia of P. polycephalum extending to dead ends and connecting the maze through the shortest distance.

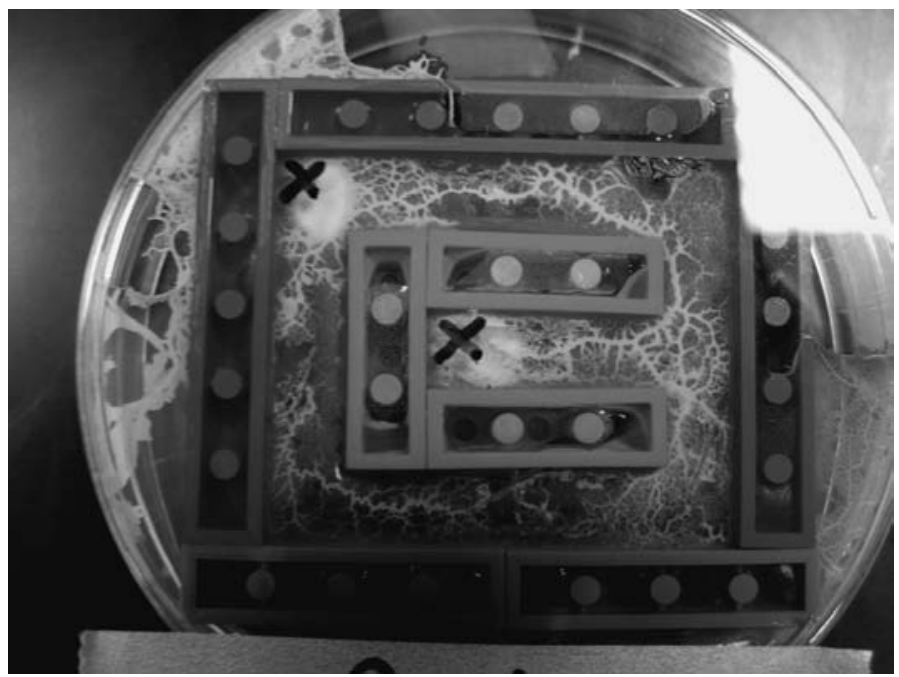

Figure 2. View of the bottom of a Petri dish showing results of a maze experiment 2 days after fresh oats were placed (on $X^{\prime}$ 's). Living plasmodium is connecting the maze through the shortest distance, with white dead plasmodia in other regions; however, in this case, the Lego blocks were not an effective barrier. Some P. polycephalum "escaped" on the left-hand side. is usually enough to guide them to the point of measuring with a ruler the distances of all the possible routes. At this point, the concept of dynamic barriers could be introduced by having students ask additional questions regarding the adaptability of the slime mold. For instance, if the shortest maze route gets "shut down" by rearrangement or addition of a new Lego barrier, then the previously longer route now becomes the shortest and students can consider whether the plasmodium responds accordingly and reinforce the new shortest route. This could be related to larger themes of unpredictability and serendipity in the movement and development of organisms to new habitats. A series of videos showing P. polycephalum growing through (and over) a Lego maze have been posted on SchoolTube at http://www.schooltube.com/user/slimemold.

Figure 3 shows the results of two of the plates used in the openinquiry experiment described in Table 1 . This set of plates shows
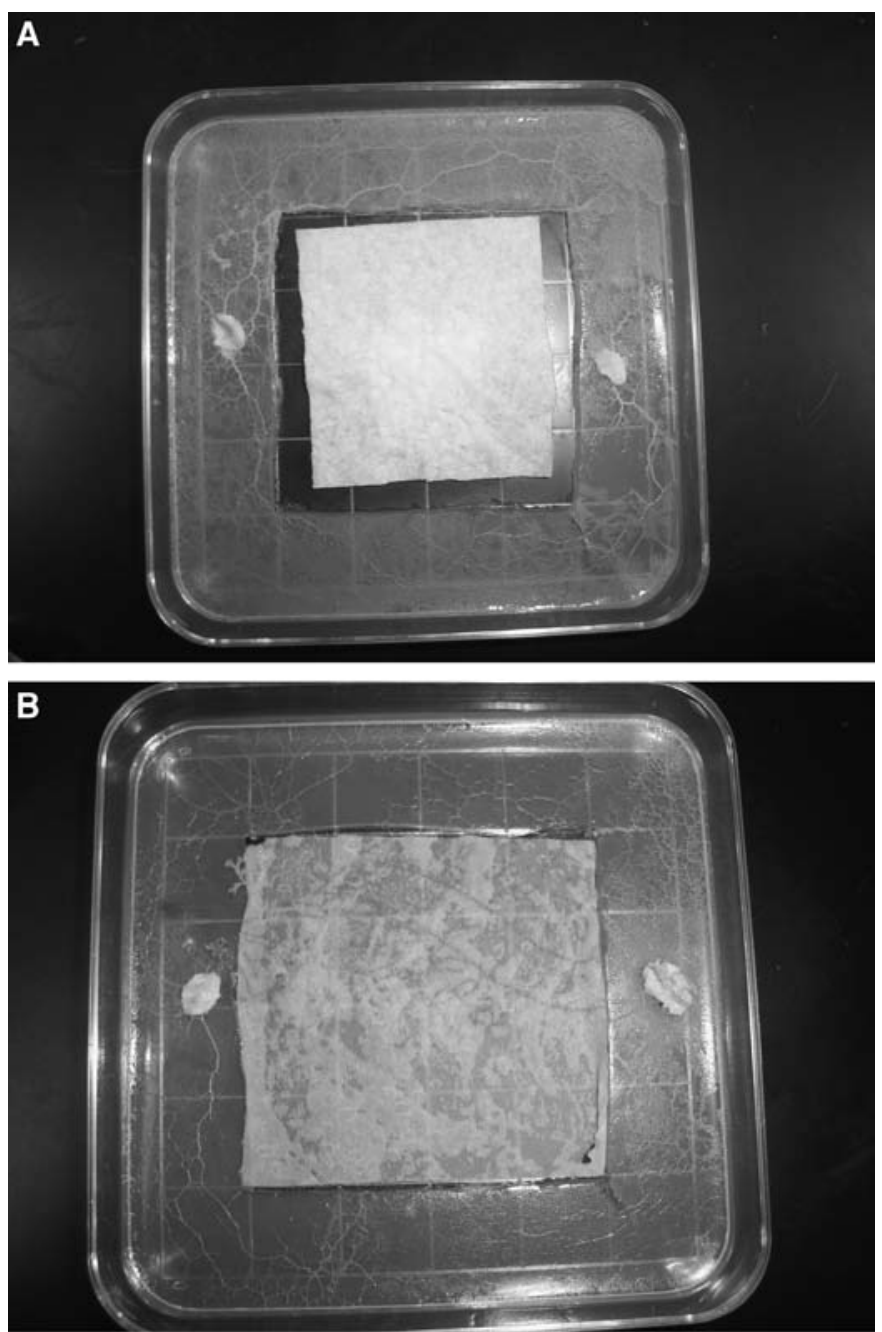

Figure 3. These images show growth of the slime mold 3 days after inoculation on plates with (A) a dry center or (B) a wet center. Students used a piece of paper towel slightly smaller than the cut area so that the paper towel would not absorb the moisture of the agar. On the wet plate, the slime mold eventually connected the two oats through the shortest distance across the wet paper towel. On the dry plate, the slime mold grew around the barrier of the dry paper towel. On both plates, areas of dead plasmodia (and places where the slime mold once grew) can be seen as white tubes. 
that lack of moisture can be an effective barrier to movement. The comparison also emphasizes the importance of controls in experimental design. The relative ease in setting up these experiments and collecting data allows students to set up multiple controls and revise their experiments as needed. For this reason, the openinquiry phase of this investigation is particularly powerful in teaching students the logic behind experimental design and the necessity of controls.

Through this unique lab experience, students learn about the importance of barriers in controlling the movement of an organism. Through their own experimentation, the students experience science as a process and gain insight into the nature of science. As opposed to a traditional, non-inquiry approach to labs, there is no right answer to this problem; there is no perfect barrier to "cage the blob" and control the growth of the plasmodium. Creating barriers that effectively control, corral, or otherwise shape the movement of organisms is challenging. Through their own experiments, students will have first-hand experience in just how challenging it can be. Students may find some barriers that work for some lab groups but not others, or other barriers that are effective in the short term but not over the long term. Because each lab group has a different experience, class presentations of experiments are a good assessment tool. Students learn from each other, and the teacher can guide class discussion as students talk through how they designed their experiment and what improvements could be made in the future. Also, when students present their data, they really have to think through how to provide evidence to support their claims about the effectiveness of a certain type of barrier.

Biology students are not often exposed to the subject of "chaos" as it relates to biology (e.g., complicated time-varying patterns; Strogatz, 1994). This lab provides a unique jumping-off point for students to look at chaos as it relates to ecology, such as predatorprey cycles and the role of transient events in ecology, such as the forming and dissolution of land bridges and other dynamic routes as they relate to global migrations.

This lab also provides an opportunity for students to have a class discussion about the nature of intelligence. It may be useful for the teacher to review Nakagaki's (2001) article arguing that P. polycephalum exhibits primitive intelligence. Thomas's (1980) article describing a hierarchy of learning abilities may also be helpful. This element of the lab is one that may appeal more to the humanities and social-science-oriented learners in the classroom. In this way, this lab experience appeals to diverse interests and learning styles (from experimental design to philosophy to playing with Legos), engaging students from different backgrounds in learning science. Students are given the freedom to explore, to be inspired by science. They may even be able to outsmart a slime mold.

\section{$\bigcirc$ Acknowledgments}

We thank the Governor's School students who enthusiastically piloted this lab and provided valuable feedback on how to make it better. Thanks also to Dr. Erin Dolan for her guidance and assistance in evaluating student learning from this project. This material is based on work supported by the National Science Foundation under grant no. 0919088 to D. Schmale and S. Ross. Any opinions, findings, and conclusions or recommendations expressed in this material are those of the authors and do not necessarily reflect the views of the National Science Foundation.

\section{References}

Adamatzky, A. (2010). Routing Physarum with repellents. European Physical Journal E, 31, 403-410.

Aylor, D.E., Schmale, D.G., III, Shields, E.J., Newcomb, M. \& Nappo, C.J. (2011). Tracking the potato late blight pathogen in the atmosphere using unmanned aerial vehicles and Lagrangian modeling. Agricultural and Forest Meteorology, 151, 251-260.

Grodowitz, M.J. (2002). United States water ballast regulations. In Zebra Mussel Information System. [Online.] Available at http://el.erdc. usace.army.mil/zebra/zmis/zmishelp/united_states_ballast_water_ regulations.htm.

Isard, S.A. \& Gage, S.H. (2001). Flow of Life in the Atmosphere: An Airscape Approach to Understanding Invasive Oróanisms. East Lansinģ, MI: Michigan State University Press.

Isard, S.A., Gage, S.H., Comtois, P. \& Russo, J.M. (2005). Principles of aerobiology applied to soybean rust as an invasive species. BioScience, $55,851-862$

Knight, W. (2006). Robot moved by a slime mould's fears. New Scientist, 13 February. Available at http://www.newscientist.com/article/dn8718robot-moved-by-a-slime-moulds-fears.html.

Latty, T. \& Beekman, M. (2010). Food quality and the risk of light exposure affect patch-choice decisions in the slime mold Physarum polycephalum. Ecology, 91, 22-27.

Lekien, F. \& Ross, S.D. (2010). The computation of finite-time Lyapunov exponents on unstructured meshes and for non-Euclidean manifolds. Chaos, 20, 017505.

Miller, G.T. \& Spoolman, S.E. (2009). Living in the Environment: Concepts, Connections, and Solutions, $16^{\text {th }}$ Ed. Belmont, CA: Brookes/Cole, Cengage Learning.

Nakagaki, T. (2001). Smart behavior of true slime mold in a labyrinth. Research in Microbiology, 152, 767-770.

Nakaģaki, T., lima, M., Ueda, T., Nishiura, Y., Saiggusa, T., Tero, A., Kobayashi, R. \& Showalter, K. (2007). Minimum-risk path finding by an adaptive amoebal network. Physical Review Letters, 99, 068104.

Nakaģaki, T., Yamada, H. \& Tóth, Á. (2000). Maze-solving by an amoeboid organism. Nature, 407, 470.

National Academy of Sciences. (1996). National Science Education Standards.Washington, D.C.: National Academy Press.

Schumann, G.L. \& D'Arcy, C.J. (2000). Late blight of potato and tomato. In The Plant Health Instructor. Available at http://www.apsnet.org/edcenter/ intropp/lessons/fungi/Oomycetes/Pages/LateBlight.aspx.

Strogatz, S.H. (1994). Nonlinear Dynamics and Chaos: With Applications in Physics, Bioloğy, Chemistry, and Enǵineerinǵ. Reading, MA: Addison-Wesley.

Tero, A., Takaģi, S., Saigusa, T., Ito, K., Bebber, D.P., Fricker, M.D. \& others. (2010). Rules for biologically inspired adaptive network design. Science, 327, 439-442.

Thomas, R.K. (1980). Evolution of intelligence: an approach to its assessment. Brain, Behavior and Evolution, 17, 454-472.

CINDY E. BOHLAND teaches biology and biotechnology at the Roanoke Valley Governor's School for Science and Technology, 2104 Grandin Road, Roanoke, VA 24015; e-mail: cbohland@rvgss.k12.va.us. DAVID G. SCHAMLE III is Associate Professor in the Department of Plant Pathology, Physiology, and Weed Science at Virģinia Tech, Blacksburģ, VA 24061-0390; e-mail: dschmale@ vt.edu. SHANE D. ROSS is Assistant Professor in the Department of Enģineering Science and Mechanics, Virģinia Tech, Blacksburģ, VA 24061-0291; e-mail: sdross@vt.edu. 